\title{
The Kinematics Analysis and Configuration Optimize of Quadruped Robot
}

\author{
Jinrong Zhang ${ }^{*}$, Chenxi Wang and Jianhua Zhang
}

Chang'an University, Xi'an Shaanxi, 710064, China

\begin{abstract}
In order to obtain the optimal structure parameters of a quadruped robot, in this paper, a four-legged mammal $(\mathrm{dog})$ is used as a model, and a quadruped robot configuration framework is set up by simplification of the model's structure. In addition, the kinematics model of this robot is established. Based on the analysis of quadruped robot's kinematics characteristics, and taking the flexibility ratio for evaluation criterion, structure parameters are optimized. And then, kinematics simulation of the robot is done in ADAMS software. The simulation results validate the rationality of configuration design of the quadruped robot.
\end{abstract}

Keywords: Quadruped robot, Kinematics, Configuration optimize, Simulation, Virtual prototype

\section{INTRODUCTION}

At present, according to robots' locomotion methods, they are divided into several categories: wheeled type, crawler-type and foot type. Wheeled type and crawler-type robots have weak obstacle climbing ability and flexibility. Walking legs of quadruped robots have multiple degrees of freedom and their landing points are scattered, so they can flexibly adjust the walking posture within the reachable scope and select reasonable support points which will provide a high obstacle avoiding and climbing ability. To achieve these advantages, quadruped robots are required of a high flexibility.

Flexibility can be improved by increasing the number of leg joints and determining a reasonable proportion of different parts of a leg. Increasing the number of leg joints to build a redundancy structure is not suitable because of the technical limitation, such as control and drive.

In this study, firstly, a structure design of quadruped robot was proposed by the use of a quadruped mammalian animal as a model. Then the kinematics analysis was completed for the robots, and proportion of different parts of a leg of the robot was optimized. Finally, the optimized structure was checked out by simulations in ADAMS.

\section{STRUCTURE DESIGN OF QUADRUPED ROBOT}

\subsection{Establishment and Simplification of Mechanism Model}

It is shown in Fig. (1) that quadruped mammal animals' leg is composed of five parts, which formed five joints by connection with the trunk. There is $1 \sim 3$ degrees in each joint, and the animal moves flexibly because of the hyperredundant degrees of freedom [1]. It's almost impossible for the quadruped bionic robot to completely imitate this kind of structure at this stage because of the complexity of control and machine. Reasonable simplification need to be completed to reduce the complexity of the robot control and guarantee flexibility $[2,3]$. As it is shown in Fig. (2), the walking leg consists of side swing joint, thigh and calf. Trunk, side swing joint, thigh and calf are respectively connected by rotational joints, each of which has one degree of freedom. The structure and parameters of the four legs are the same and they are symmetrically distributed in the coordinate plane of the trunk.

\subsection{Confirmation of Degrees of Freedom}

While a quadruped robot is walking, the movement of the walking leg can be divided into two states: swing state and support state. In swing state, the walking leg performed as an open chain structure of connected with link rod, and the freedom degree of the walking leg is equal to the number of joints. In support state, trunk is a central component of the parallel mechanism, and the ground can be used as frame of parallel mechanism. There is the enough friction between the support foot and the ground that there is no slip between them, and it can be viewed as a spherical joint with the ground [4]. If the number of legs which are in support state is $n(n \leq 4)$, then the model is a spatial multi-loop parallel mechanism with $n$ branches, and the number of degrees of freedom can be calculated by this formula:

$M=6(m-g-1)+\sum_{i=1}^{4} f_{i}$

Where, 


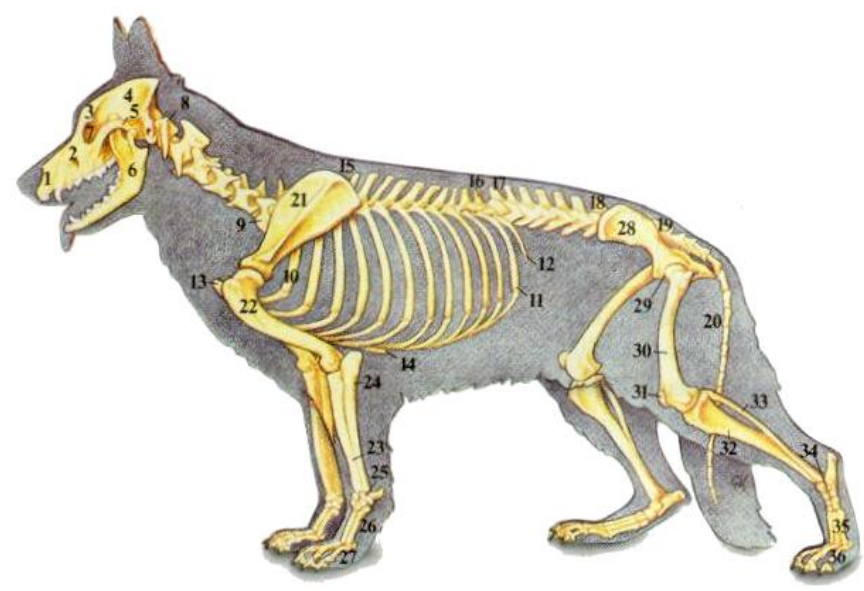

Fig. (1). Bones of dog.

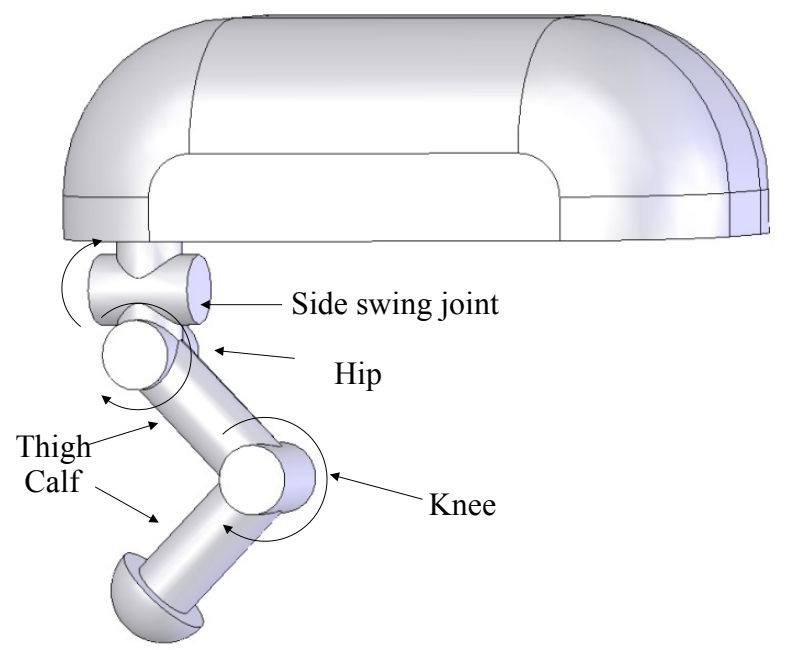

Fig. (2). Simplified model of a leg.

Table 1. Parameters of connecting rods and joints of a leg.

\begin{tabular}{|c|c|c|c|c|c|}
\hline Number of Connecting Rod & Angel of Joint & $\alpha$ & $a$ & $d$ & $\cos \alpha$ \\
\hline \hline 1 & $\theta_{1}$ & $90^{\circ}$ & $l_{1}$ & 0 & 0 \\
\hline 2 & $\theta_{2}$ & $0^{\circ}$ & $l_{2}$ & 0 & 1 \\
\hline 3 & $\theta_{3}$ & $0^{\circ}$ & $l_{3}$ & 0 & 1 \\
\hline
\end{tabular}

$m$ : Total number of components, $m=3 n+2$;

$g:$ Number of joints, $g=4 n$;

$\sum_{i=1}^{4} f_{i}:$ The sum of degrees of the joints.

After corresponding parameters were substituted in formula 1, the total number of freedom degrees $(M)$ can get where $M=6$. So the total number of degrees of freedom has nothing to do with the number of the support leg. Therefore, no matter what kind of gait the robot walked with, its body could realize any position and posture in a certain range.

\section{KINEMATIC ANALYSIS}

Each walking leg of the quadruped robot can be regarded as a series open kinematic chain, and all the joint connecting rods were series connected together by revolute pairs. D-H method was used in this paper to get position of the reference points in the end of a quadruped robot's swing leg, as is shown in Table $\mathbf{1}$ and Fig. (3). 


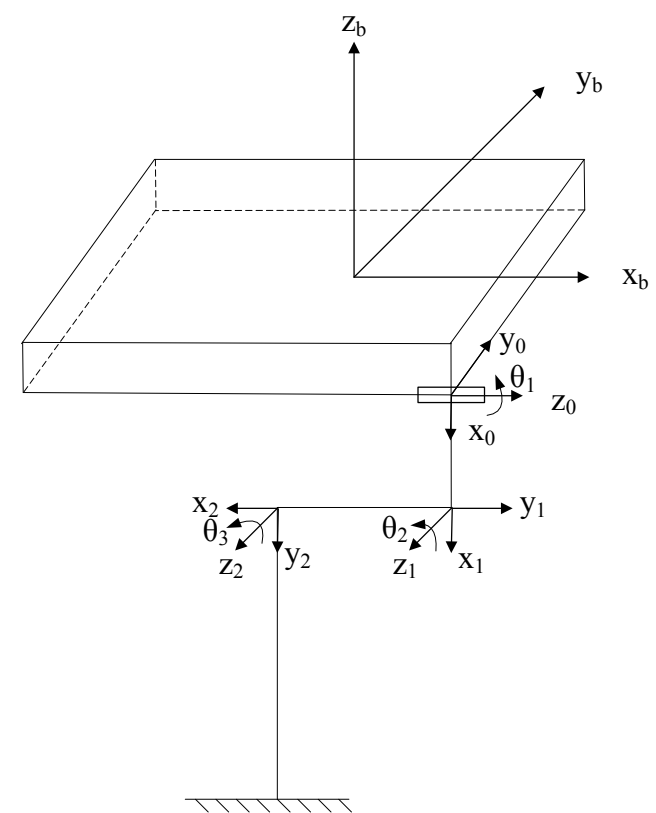

Fig. (3). D-H coordinate system of a leg.

(1) Establish a coordinate system $\left(X_{b}, Y_{b}, Z_{b}\right)$ of the body, and the origin of it was the initial position of the body's center of mass. The positive direction of the $X_{b}$ axis was the heading direction of the robot, while the positive direction of the $Z_{b}$ axis was the opposite direction of the gravity. Then the direction of the $Y_{b}$ axis can be defined by the right hand rule.

(2) Establish a coordinate system on every connecting rod. The relative position and attitude of these coordinate systems can be described by homogeneous transformation

(3) Establish a coordinate system of the side swing joint, in which the coordinate of the origin point $O_{0}$ was $(a, b, c)$.

The transformation matrix of the body coordinate system and $\{0\}$ is $A b 0$, it was shown as follows:

$$
A_{b 0}=\left[\begin{array}{cccc}
0 & 0 & 1 & a \\
0 & 1 & 0 & b \\
-1 & 0 & 0 & c \\
0 & 0 & 0 & 1
\end{array}\right]
$$

The homogeneous transformation matrix between adjacent the coordinate system of legs is $A_{i-1, i}$ :

$$
A_{i-1, i}=\left[\begin{array}{cccc}
c \theta_{i} & s \theta_{i} c \alpha_{i} & s \theta_{i} s \alpha_{i} & a_{i} c \theta_{i} \\
s \theta_{i} & c \theta_{i} c \alpha_{i} & -c \theta_{i} s \alpha_{i} & a_{i} s \theta_{i} \\
0 & s \alpha_{i} & c \alpha_{i} & d_{i} \\
0 & 0 & 0 & 1
\end{array}\right]
$$

Where,

$$
c \theta_{i}=\cos \theta_{i}, s \theta_{i}=\sin \theta_{i}, c \alpha_{i}=\cos \alpha_{i}, s \alpha_{i}=\sin \alpha_{i}
$$

The conversion matrix of the body coordinate system and the coordinate fixed to the end of foot is $T_{b 3}$ :

$T_{b 3}=A_{b 0} A_{01} A_{12} A_{23}=\left(\begin{array}{cccc} & & & P_{x} \\ & R & & P_{y} \\ & & & P_{z} \\ 0 & 0 & 0 & 1\end{array}\right)$

$R$ is the attitude matrix of the end of foot.

The coordinates of the end of the foot was $P_{x} 、 P_{y} 、 P_{z}$, and they are defined as follows according to formulate (4):

$$
\begin{aligned}
& P_{x}=l_{3} s_{23}+l_{2} s_{2}+a \\
& P_{y}=l_{3} s_{1} c_{23}+l_{2} s_{1} c_{2}+l_{1} s_{1}+b \\
& P_{z}=-l_{3} c_{1} c_{23}-l_{2} c_{1} c_{2}-l_{1} c_{1}+c
\end{aligned}
$$

Where,

$$
\begin{aligned}
& s_{i j}=\sin \theta_{i} \cos \theta_{j}+\cos \theta_{i} \sin \theta_{j}, \\
& c_{i j}=\cos \theta_{i} \cos \theta_{j}-\sin \theta_{i} \sin \theta_{j}, \\
& s_{i}=\sin \theta_{i}, c_{i}=\cos \theta_{i} .
\end{aligned}
$$

In the posture control and trajectory planning of the robot, real-time targets of the joints can be acquired by the 


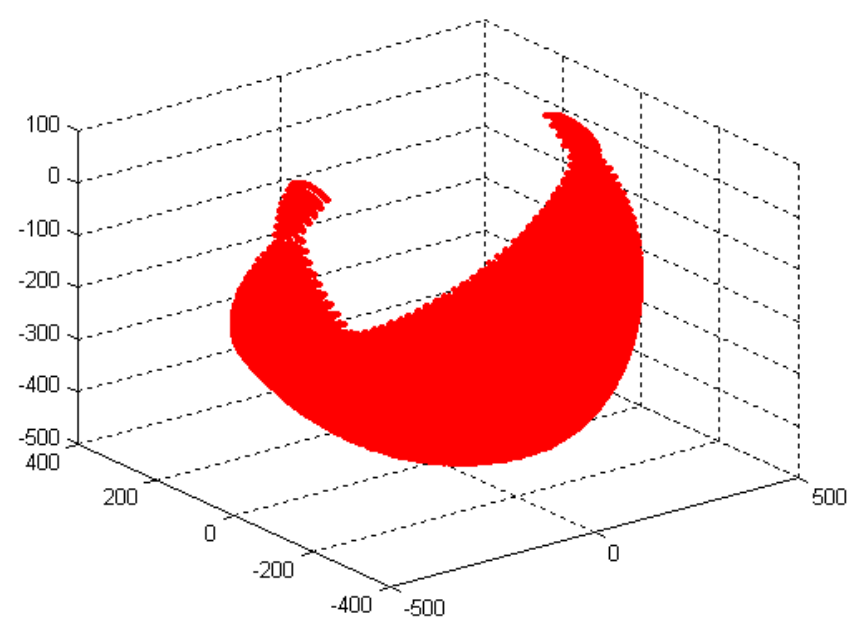

Fig. (4). Cloud map of the foot's workspace of the quadruped robot's swing leg.

inverse kinematic solution of the walking leg in the solving progress of motivation, and they can be used as inputs for each joint actuator to control the rotation [5].

$\theta_{1}=\operatorname{tg}^{-1}\left(\frac{p_{y}-b}{-p_{z}+c}\right)$

$\theta_{2}=\operatorname{tg}^{-1}\left[\frac{D^{2}+\left(p_{x}-a\right)^{2}+l_{2}^{2}-l_{3}^{2}}{\sqrt{4 l_{2}^{2}\left[D^{2}+\left(p_{x}-a\right)^{2}\right]^{2}-\left[D^{2}+\left(p_{x}-a\right)^{2}+l_{2}^{2}-l_{3}^{2}\right]^{2}}}\right]-\operatorname{tg}^{-1} \frac{D}{p_{x}-a}$

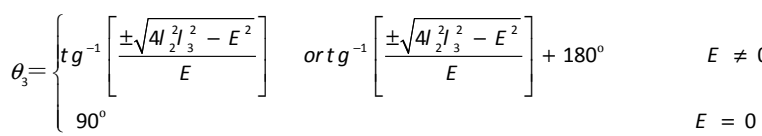

Where,

$D=c_{1}\left(-p_{z}+c\right)+s_{1}\left(p_{y}-b\right)-l_{1}, E=D^{2}+\left(p_{x}-a\right)^{2}-l_{2}^{2}-l_{3}^{2}$

\section{OPTIMIZATION OF STRUCTURE PARAMETERS AND SIMULATION}

\subsection{Optimization of Structure Parameters}

The quadruped robot can choose a foothold in a larger space because of the flexibility of the leg. When walking in the static gait, it can also ensure the trunk to change posture in a free and wide range in the spatial parallel mechanism consisted of the trunk, supporting leg and the ground in order to adapt to the ground conditions [4]. In the perspective of mechanism, a leg is equivalent to a series manipulator in the mechanism, so space points of foot, a collection of reachable special positions, can be used as the evaluation indicator of its flexibility. In the process of the parameters analysis and optimization of the leg structure, the goal is to reach the feet's biggest activity space with the best proportion of femur length.
Due to the complexity of the structure parameters of quadruped robot, virtual prototype technology can be used to simplify the process. The following assumptions and parameter setting should be made when establishing the virtual prototype of quadruped robot

(1) Every components of quadruped robot are rigid bodies.

(2) Considering the structure and motion characteristics of quadruped robot, a rotating pair and a driving were added in every leg joint and ball joints were added between the foot and the ground to meet the need of the posture transformation. In theory, the greater the intersection angle is, the higher flexibility robots has, which is mainly affected by structural factors. However in the actual design, the intersection angle only can be as large as possible while ensuring the compact structure size. Where, $\theta_{1} \leq 30^{\circ}, \theta_{2} \leq 70^{\circ}, \theta_{3} \leq 80^{\circ}$.

(3) The control law of the joint driving can be acquired by the inverse kinematics which is define as formulate (8) $\sim(10)$.

(4) Crash forces and sensors should be added to avoid the interference among the trunk, legs and the ground.

(5) The design variables are the length of side swing joint, thigh and calf $\left(l_{1}, l_{2}\right.$ and $\left.l_{3}\right)$, restrain is $l_{1}+l_{2}+l_{2}=470 \mathrm{~mm}$, and the optimization target is the maximum coverage area of the foot.

Through the repeated calculation, the maximum space of the foot can be obtained when $l_{1}: l_{2}: l_{3}=1: 3: 3.6$, that means $l_{1}=62 \mathrm{~mm}, l_{2}=185 \mathrm{~mm}, l_{3}=223 \mathrm{~mm}$. The cloud map of the foot's workspace of the quadruped robot's swing leg was shown in Fig. (4) when the values of $\left(l_{1}, l_{2}, l_{3}\right)$ were substituted in formula (5)-(7). 


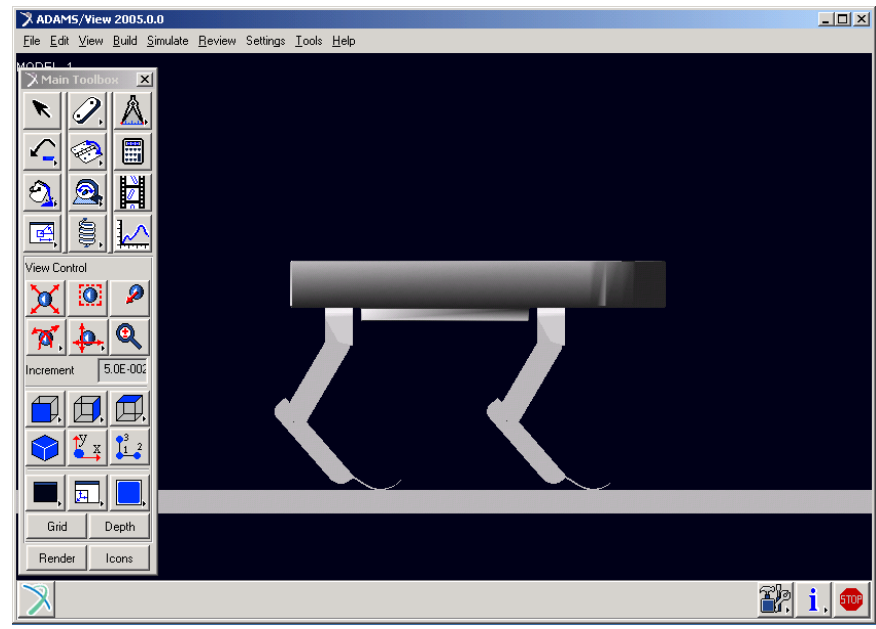

Fig. (5). Simulation model.

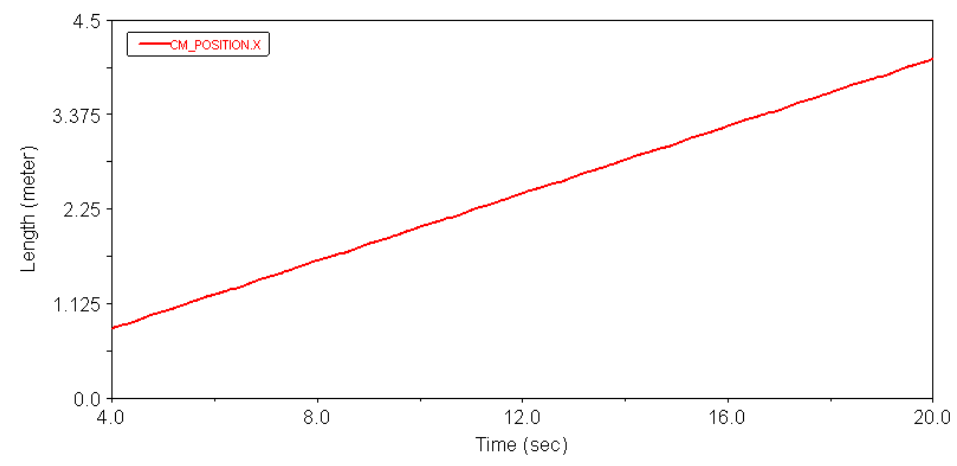

Fig. (6). Displacement of mass center in $\mathrm{X}$ direction

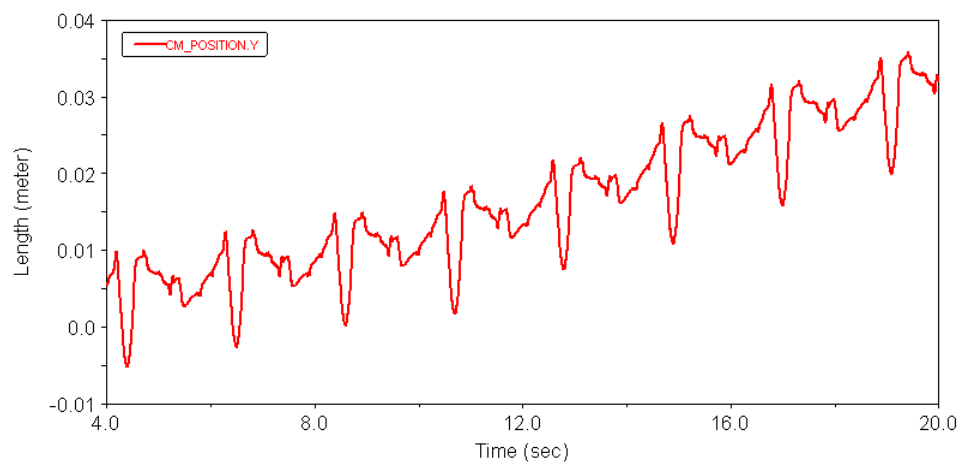

Fig. (7). Displacement of mass center in $\mathrm{Y}$ direction.

\subsection{Simulation}

ADAMS is the most widely used simulation software for mechanical system in the world. It can build a digital virtual prototyping to simulate the physical prototype based on the actual movement. It also can improve and optimize the design conveniently, test and evaluate the results of designs, simplify the process of development, shorten the development cycle, reduce the cost and improve the quality of design. Virtual prototype of quadruped robot is shown in Fig. (5).
In the simulation, optimized parameters were used in the model. The simulation results are shown in Figs. (6-8).

The position changes of robot's center of mass were shown in the figure above: the shifting of the way forward was $3200 \mathrm{~mm}$, with the speed of $200 \mathrm{~mm} / \mathrm{s}$. there was a deviation to the right of $25 \mathrm{~mm}$, basically met the requirement of walking in a straight line. The fluctuation rate of the center of mass in vertical direction was $0.79 \%$, for the reason that the wave amplitude was $3.95 \mathrm{~mm}$ and the height of robot was $500 \mathrm{~mm}$ 


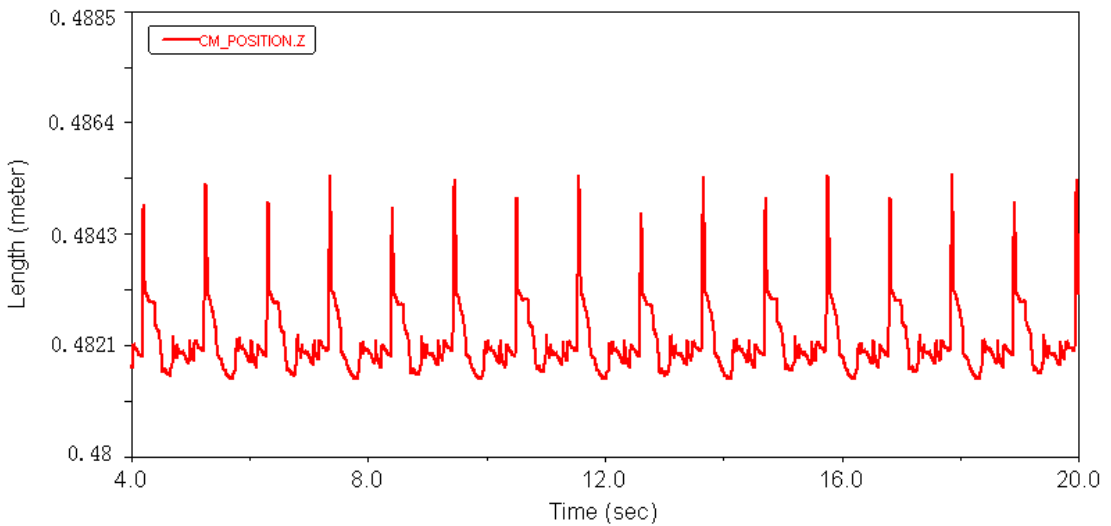

Fig. (8). Displacement of mass center in $\mathrm{Z}$ direction.

The analysis about the speed, linearity and stability of quadruped robot can validate the rationality of the optimized structure design.

\section{CONCLUSION}

(1) A simplified structure model of the quadruped robot was built and initial parameters were identified by the observation and measurement about the quadruped mammalian animal. Based on the kinetic analysis of a quadruped robot, normal solution and inverse solution of walking leg can be obtained, which can lay the foundation for the subsequent trajectory planning and simulation.

(2) Dynamic simulation optimized the initial structure parameters with the index of flexibility. The walking process of quadruped robot was observed on the basis of visual simulation by the software, fully validating the plan of structure design

\section{CONFLICT OF INTEREST}

The authors confirm that this article content has no conflicts of interest.

\section{ACKNOWLEDGEMENTS}

Declared none.

\section{REFERENCES}

[1] Thanhtam Ho, Sunghac Choi, Sangyoon Lee. Development of a Biomimetic Quadruped Robot. Journal of Bionic Engineering, 2007, 4, 193-199.

[2] Playter R, BuehlerM, RaibertM. BigDog. Proceedings of SPIE, Unmanned Systems Technology VIII. 2006,6230

[3] Rebula JR, et al. A controller for the little dog quadruped walking on rough terrain. Proceedings of the IEEE International Conference on Robotics and Automation (ICRA), 2007, 1467-1473

[4] Mingyang Wu, Qingxin Meng, Monan Wang. Research on Designing of Crab-liked Robot System. Forestry Machinery \& Woodworking Equipment. 2005, 4.

[5] Monan Wang, Liquan Wang, Qingxin Meng, Peng Yuan, Yanshuang Yu. Dynamic modeling and simulation of biomimetic crablike robot with ADAMS. Journal of Harbin Engineering University. 2003, 4 .

(C) Zhang et al.; Licensee Bentham Open.

This is an open access article licensed under the terms of the Creative Commons Attribution Non-Commercial License (http://creativecommons.org/licenses/by-nc/3.0/) which permits unrestricted, non-commercial use, distribution and reproduction in any medium, provided the work is properly cited. 